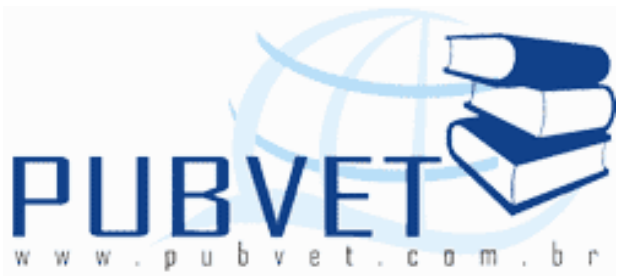

PUBVET, Publicações em Medicina Veterinária e Zootecnia.

\title{
Comparação entre as principais técnicas de análise da concentração espermática em exames andrológicos de touros a campo
}

\section{Cristiano Pereira Barbosa ${ }^{1}$; Wagner Carlos de Oliveira ${ }^{2}$; Waldir Rosa Ribeiro Júnior $^{3}$}

${ }^{1}$ Doutor, Prof. do Curso de Medicina Veterinária, Universidade de Uberaba (UNIUBE) / e-mail: crispb@netsite.com.br

${ }^{2}$ Médico veterinário, coordenador de programas de IATF da ABS-Pecplan no oeste do Estado da Bahia.

${ }^{3}$ Médico veterinário autônomo.

\section{Resumo}

A concentração espermática em exames andrológicos de touros possui a maior variabilidade em termos de disponibilidade de técnicas. Os três métodos mais utilizados atualmente como rotina são, a câmara de Neubauer, o espectrofotômetro e o espermiodensímetro, que apresentam grandes diferenças em termos de agilidade e velocidade de execução, além de custo de aquisição. Este trabalho possuiu o objetivo de comparar os resultados destas três técnicas entre si, para a melhor indicação a campo. Para isto foram utilizadas 143 amostras de sêmen de touros, as quais foram submetidas a análise pelas três técnicas entre agosto de 2007 e maio de 2008, no município de Uberaba, MG, sendo os resultados analisados pelo teste de análise estatística descritiva, teste de Correlação de Pearson e teste de diferença entre 
BARBOSA, C.P., OLIVEIRA, W.C. e RIBEIRO JÚNIOR, W.R. Comparação entre as principais técnicas de análise da concentração espermática em exames andrológicos de touros a campo. PUBVET, Londrina, V. 5, N. 16, Ed. 163, Art. 1100, 2011.

médias pelo teste $t$ de Student. Os resultados das médias encontrados foram aproximadamente $431 \times 10^{6}, 508 \times 10^{6}$ e $398 \times 10^{6}$, em espermatozóides por mililitro de sêmen, respectivamente, para as técnicas de espermiodensímetro, câmara de Neubauer e espectrofotômetro. Houve ainda alta correlação entre as três técnicas $(r=0,7904 ; 0,9143 ; 0,7099)$ em nível de significância de $1 \%$ $(p<0,01)$. No entanto, houve diferença significativa apenas entre os valores médios obtidos pela câmara de Neubauer e espectrofotômetro $(p<0,05)$, evidenciando-se a técnica de espermiodensímetro como semelhante às demais em seus valores $(p>0,05)$. Concluiu-se que o espermiodensímetro se bem padronizado, pode ser utilizado na avaliação da concentração espermática em exames andrológicos de touros à campo, devido ser de mais rápida execução que a câmara de Neubauer e de menor custo que o espectrofotômetro, com a mesma segurança nos resultados.

Palavras-chave: andrológico; touros; Neubauer; espermiodensímetro; espectrofotômetro.

\title{
Comparison between the main techniques of sperm concentration analysis in bulls breeding soundness exams
}

\begin{abstract}
The sperm concentration in breeding soundness exams of bulls is the most variable in terms of amount of techniques. The three methods more used currently as routine are: the Neubauer's chamber, spectrophotometry and sperm densitometer. The three techniques show differences in velocity of execution and costs. This work had the objective to compare the three techniques. In this study were used 143 bulls semen samples. These samples were collected in Uberaba's farms from August 2007 till May 2008. The results were analyzed by: the descriptive statistics analysis, the Pearson's Correlation Test and test of difference between averages for $t$ test of Student. The values of averages were approximately $431 \times 10^{6}, 508 \times 10^{6}$ and $398 \times 10^{6}$ in spermatozoa for milliliter of semen, respectively for sperm densitometer,
\end{abstract}


BARBOSA, C.P., OLIVEIRA, W.C. e RIBEIRO JÚNIOR, W.R. Comparação entre as principais técnicas de análise da concentração espermática em exames andrológicos de touros a campo.

PUBVET, Londrina, V. 5, N. 16, Ed. 163, Art. 1100, 2011.

Neubauer's chamber and spectrophotometry. The analysis found a high correlation between the three techniques ( $r=0,7904 ; 0,9143 ; 0,7099)$ $(p<0,01)$. However, it was observed a significant difference between the average values of the Neubauer's chamber and spectrophotometry $(p<0,05)$ but it was not found any difference between the sperm densitometer and the others $(p>0,05)$. It was concluded that the sperm densitometer technique can be used in the evaluation of sperm concentration in bulls breeding soundness exams in the field, because it is faster that the Neubauer's chamber and lower cost comparing to the spectrophotometry and with the same security on the results.

Keywords: breeding soundness exam; bulls; Neubauer's chamber; sperm densitometer; spectrophotometry.

\section{INTRODUÇÃO}

O Brasil possui atualmente em torno de 205 milhões de cabeças em seu rebanho bovino sendo que deste total, aproximadamente 60 milhões de fêmeas estão em idade reprodutiva (BRASIL, 2009). Para atender a este número de animais são necessários a utilização de machos reprodutores em situação de monta natural ou então em inseminação artificial (I.A.), em um número aproximado de 1,7 milhão de touros. No Brasil, a I.A. tem atingido apenas 7\% do rebanho total (ASBIA, 2009), demonstrando a grande necessidade de touros para servir a estas fêmeas.

Para que haja um bom desempenho reprodutivo eles devem ter a habilidade de detectar o cio, capacidade para realizar o salto ou a monta propriamente dita e disposição ou alta libido para servir as fêmeas de forma eficiente. Porém entre todas estas características, sob o ponto de vista reprodutivo, a mais importante para o macho reprodutor é a fertilidade, ou a capacidade de fecundar a fêmea. Portanto a fertilidade do touro envolve a produção de espermatozóides viáveis, com capacidade de locomoção e em concentração satisfatória. 
BARBOSA, C.P., OLIVEIRA, W.C. e RIBEIRO JÚNIOR, W.R. Comparação entre as principais técnicas de análise da concentração espermática em exames andrológicos de touros a campo.

PUBVET, Londrina, V. 5, N. 16, Ed. 163, Art. 1100, 2011.

De acordo com Pimentel (1998) o principal objetivo do exame andrológico é o de se fazer uma estimativa da fertilidade potencial do touro e, em segundo lugar, identificar anormalidades no trato genital ou no comportamento sexual que possam comprometer a fertilidade. Em terceiro lugar, deve-se observar seu mérito genético para o fim a que se destina. Um touro pode se apresentar sub-fértil quando possui problemas no sêmen, não consegue executar o ato sexual ou quando é portador de doenças venéreas. A importância do exame andrológico reside no fato de um touro servir, no mínimo, 25 vacas por temporada em regime de monta natural, até milhares, através de inseminação artificial. Neste caso, reveste-se de importância a avaliação de seu mérito genético.

A análise da concentração espermática é um método de grande importância para a avaliação da aptidão de um touro à reprodução. Os métodos utilizados com mais freqüência atualmente são a câmara de Neubauer, o espectrofotômetro e o espermiodensímetro. A utilização destas três técnicas a campo tem revelado que não há ainda uma que se sobressaia em todos os aspectos (PIMENTEL E DESCHAMPS, 1995).

De acordo com Tomlinson et al. (2001) a avaliação da concentração espermática pela câmara de Neubauer é considerada como procedimento padrão na andrologia.

A câmara de Neubauer é de alta acurácia, com menor custo, porém de execução mais demorada e trabalhosa. O espectrofotômetro é altamente utilizado em centrais de inseminação artificial por ser de rápida execução porém seu custo é alto, enquanto o espermiodensímetro apresenta um custo intermediário, uma execução rápida, porém ainda requer padronização para evitar resultados equivocados no exame (FARRELL et al., 1998).

A espectrofotometria é segundo Farrell et al. (1998) a técnica mais rápida e precisa de todas as convencionais, para a análise da concentração espermática em exames de qualidade de sêmen, porém técnicas modernas como análise espermática monitorada por computador, tem surgido como 
BARBOSA, C.P., OLIVEIRA, W.C. e RIBEIRO JÚNIOR, W.R. Comparação entre as principais técnicas de análise da concentração espermática em exames andrológicos de touros a campo.

PUBVET, Londrina, V. 5, N. 16, Ed. 163, Art. 1100, 2011.

métodos de avaliação completa inclusive da motilidade, apesar de se apresentar com custo mais elevado.

Palmer e Barth (2003) utilizaram a técnica de avaliação da concentração espermática em touros pelo densitômetro, cuja capacidade é demonstrar por um sensor óptico, através de deflecção de luz, os movimentos causados pelos espermatozóides. Ao compararem este procedimento com o convencional, de utilização de técnicas microscópicas, que avaliam a porcentagem de espermatozóides móveis e principalmente sua concentração no líquido seminal, eles concluíram que o densitômetro não é um bom substituto das referidas tecnologias, por apresentar característica mais subjetiva.

A técnica de determinação da concentração espermática pela utilização do espermiodensímetro de Karras é bem difundida na suinocultura a fim de se quantificar $o$ número de espermatozóides presentes no ejaculado e, posteriormente, o número de doses inseminantes a serem produzidas (BRUNO et al., 2003). Esta técnica por ser de execução mais rápida é às vezes utilizada a campo em exames andrológicos de touros, principalmente em larga escala de trabalho, definindo-se a concentração espermática por um método subjetivo de visualização da densidade do volume ejaculado.

Segundo Christensen et al. (2004) as técnicas rotineiras de determinação da concentração espermática, como o espectrofotômetro e a contagem eletrônica de células, são os métodos mais rápidos e de utilização mais frequentes quando há condições de infra-estrutura. Porém novos estudos têm sido feitos com o emprego de citometria de fluxo, visando não só a análise da concentração de espermatozóides no sêmen como suas viabilidades.

O objetivo deste trabalho foi comparar três técnicas de avaliação da concentração espermática como câmara de Neubauer, espectrofotometria e espermiodensitometria, em exames andrológicos de bovinos, a fim de se definir a mais acurada e acessível para a rotina de campo. 
BARBOSA, C.P., OLIVEIRA, W.C. e RIBEIRO JÚNIOR, W.R. Comparação entre as principais técnicas de análise da concentração espermática em exames andrológicos de touros a campo.

PUBVET, Londrina, V. 5, N. 16, Ed. 163, Art. 1100, 2011.

\section{MATERIAL E MÉTODOS}

Foram utilizados 143 touros localizados em propriedades do município de Uberaba, Minas Gerais, no período de agosto de 2007 a maio de 2008, para serem realizadas as determinações de suas concentrações espermáticas. As amostras de sêmen foram coletadas a campo e em seguida encaminhadas para o laboratório de análises do Hospital Veterinário de Uberaba, onde foram realizadas as técnicas de determinação da concentração espermática com utilização de espermiodensímetro e câmara de Neubauer. Uma fração de cada amostra foi encaminhada para o laboratório de análises da central de coleta de sêmen Nova Índia, onde foi realizada a técnica de determinação da concentração espermática por espectrofotometria. Para cada touro foram realizados os três testes, do mesmo ejaculado, a fim de comparação entre eles. A execução destas três técnicas acima citadas foi a seguinte:

Para o espermiodensímetro, era colocado $10 \mathrm{~mL}$ de solução fisiológica ( $\mathrm{NaCl}$ a $0,9 \%$ ) em um recipiente graduado, próprio do Kit. Adicionava-se frações de 0,1 mL de sêmen até que a solução atingisse turbidez (densidade) mínima sobre um fundo branco, suficiente para leitura. $O$ valor da concentração espermática era então interpretado em tabela própria do material.

Para a espectrofotometria era feita a leitura da concentração em transmitância $(T)$ na faixa de $550 \mathrm{~nm}$ de comprimento de onda em aparelho de espectrofotômetro e, em seguida, comparava-se o resultado numa tabela previamente preparada, através de equação de regressão de uma amostra de sêmen de concentração conhecida.

Já para o uso da Câmara de Neubauer, foram diluídos $20 \mu \mathrm{L}$ de sêmen em $4 \mathrm{~mL}$ de solução de formol salino. Contavam-se 5 quadrados de cada lado da câmara, em diagonal, sem considerar os espermatozóides cujas cabeças estivessem sobre as bordas laterais esquerdas e inferior. O total era multiplicado por 10.000 .000 e obtinha-se a concentração em $1 \mathrm{~mL}$. 
BARBOSA, C.P., OLIVEIRA, W.C. e RIBEIRO JÚNIOR, W.R. Comparação entre as principais técnicas de análise da concentração espermática em exames andrológicos de touros a campo.

PUBVET, Londrina, V. 5, N. 16, Ed. 163, Art. 1100, 2011.

As análises estatísticas foram feitas pelo teste de análise estatística descritiva, teste de Correlação de Pearson e teste de diferença entre médias pelo teste $t$ de Student (AYRES et al., 2000).

\section{RESULTADOS}

A Tabela 1 demonstra os valores das médias e desvios padrões encontrados para as três técnicas analisadas. Eles foram aproximadamente $431 \times 10^{6}, 508 \times 10^{6}$ e $398 \times 10^{6}$, em espermatozóides por mililitro de sêmen, respectivamente, para as técnicas de espermiodensímetro, câmara de Neubauer e espectrofotômetro.

Tabela 1 - Médias e desvios padrões das três técnicas de análise da concentração espermática em touros.

\begin{tabular}{cccc}
\hline variáveis & $\mathrm{n}$ & média & $\mathrm{dp}$ \\
\hline ed & 143 & $431,6294 \mathrm{ab}$ & 291,5181 \\
cn & 143 & $508,3636 \mathrm{a}$ & 430,5076 \\
ef & 143 & $398,7762 \mathrm{~b}$ & 272,6464 \\
\hline ed = espermiodensímetro; cn = câmara de Neubauer; ef = espectrofotômetro; \\
dp = desvio padrão.
\end{tabular}

Letras diferentes na coluna indicam diferença estatística $(p<0,05)$.

Quanto às análises de médias, o desvio padrão foi bem maior para o teste com a câmara de Neubauer $\left(430 \times 10^{6}\right)$ do que para o espermiodensímetro $\left(291 \times 10^{6}\right)$ e para o espectrofotômetro $\left(272 \times 10^{6}\right)$, refletindo a grande diferença entre os valores mínimos e máximos encontrados pela referida técnica.

De acordo com a Tabela 2 houve correlação entre as três técnicas de análise da concentração espermática em nível de significância de $1 \%$ $(p<0,01)$. 
BARBOSA, C.P., OLIVEIRA, W.C. e RIBEIRO JÚNIOR, W.R. Comparação entre as principais técnicas de análise da concentração espermática em exames andrológicos de touros a campo.

PUBVET, Londrina, V. 5, N. 16, Ed. 163, Art. 1100, 2011.

Tabela 2 - Correlação e valores de t e de p entre as três técnicas de análise da concentração espermática em touros.

\begin{tabular}{ccccc}
\hline variáveis & $\mathrm{r}$ & $\mathrm{t}$ & $\mathrm{G} . \mathrm{L}$. & $\mathrm{p}$ \\
\hline edxcn & 0,7904 & $-1,76489$ & 284 & 0,078656 \\
edxef & 0,9143 & 0,98426 & 284 & 0,325824 \\
cnxef & 0,7099 & 2,571671 & 284 & 0,010630
\end{tabular}

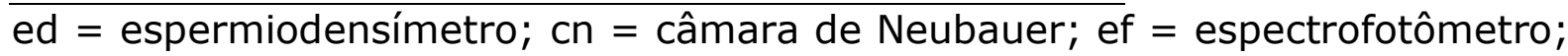
$r=$ correlação; G.L. = graus de liberdade.

Ao analisar a comparação entre as médias pelo teste $t$ de Student permitiu-se verificar diferença significativa apenas entre os valores médios obtidos pela câmara de Neubauer e espectrofotômetro $(p<0,05)$. No entanto a técnica de espermiodensímetro quando comparada às outras duas, não apresentou diferença estatística neste nível de significância.

\section{DISCUSSÃO}

Bruno et al. (2003) compararam em suínos, as técnicas de espermiodensímetro e espectrofotômetro com a câmara de Neubauer, para avaliação da acurácia, precisão e robustez destas técnicas, na análise da concentração espermática e estimativa do número de doses inseminantes, que poderiam ser produzidas por cada ejaculado. Estes autores concluíram que, em comparação com a câmara de Neubauer, que é o método mais acurado, o espectrofotômetro produziu resultados que subestimam a concentração, enquanto o espermiodensímetro produziu resultados que a superestimam, além do primeiro ter sido mais acurado que o segundo. Também eles relataram que há uma grande influência do volume total ejaculado sobre os resultados tanto do espermiodensímetro quanto do espectrofotômetro, em relação à técnica de câmara de Neubauer. Na análise destes resultados o atual trabalho diferencia dos resultados obtidos pelos autores acima citados, pois os 
BARBOSA, C.P., OLIVEIRA, W.C. e RIBEIRO JÚNIOR, W.R. Comparação entre as principais técnicas de análise da concentração espermática em exames andrológicos de touros a campo.

PUBVET, Londrina, V. 5, N. 16, Ed. 163, Art. 1100, 2011.

valores médios encontrados para espermiodensímetro foram menores do que os encontrados para câmara de Neubauer. Já os valores médios encontrados entre espectrofotômetro e câmara de Neubauer corroboram com os resultados obtidos por aqueles.

Este trabalho também discorda de Vianna et al. (2004) que relataram que o espermiodensímetro superestimou os dados padronizados pela câmara de Neubauer, mas concorda quando relatam que o espectrofotômetro subestimou.

Murgas et al. (2010) descreveram que tanto as técnicas de mensuração da concentração espermática pelo espermiodensímetro quanto pelo espectrofotômetro superestimam os valores em relação à câmara de Neubauer, sendo que neste atual trabalho ambos tiveram valores numéricos abaixo da câmara de Neubauer.

Madeira et al. (2006) concluíram que o método de avaliação da concentração espermática pelo espectrofotômetro foi o que mais se aproximou do método padrão que é a câmara de Neubauer e que os métodos de avaliação pelo aspecto do sêmen e pelo espermiodensímetro são subjetivos e não devem ser recomendados para suínos por superestimarem a dose inseminante. Neste trabalho o espermiodensímetro foi o método semelhante estatisticamente em relação à câmara de Neubauer.

Deve-se ressaltar que estes autores aqui comparados trabalharam com suínos e não com bovinos.

Quanto à adoção do método de contagem pela câmara de Neubauer, como método de referência para comparação entre as três técnicas, Sukcharoen et al. (1994) afirmam que esta técnica é considerada como procedimento padrão pela World Health Organization, o que justifica a tendência desta comparação neste trabalho.

Os valores das concentrações espermáticas obtidos pela câmara de Neubauer tendem a sofrer, segundo Sukcharoen et al. (1994), maior discrepância quando comparado com outras técnicas, principalmente para amostra com baixas concentrações, ou seja menores que $40 \times 10^{6}$ 
BARBOSA, C.P., OLIVEIRA, W.C. e RIBEIRO JÚNIOR, W.R. Comparação entre as principais técnicas de análise da concentração espermática em exames andrológicos de touros a campo.

PUBVET, Londrina, V. 5, N. 16, Ed. 163, Art. 1100, 2011.

espermatozóides/mL. Por outro lado Tomlinson et al. (2001) defendem que esta discrepância é menos pronunciada nos valores das amostras de baixas concentrações, sendo mais evidente nas amostras com concentrações próximas das normais. Estes fatos denotam que a grande variação nos resultados encontrados por outros autores, são reforçados neste trabalho onde os valores das concentrações espermáticas analisados por três métodos, demonstram diferenças apenas entre os valores obtidos pelas técnicas de câmara de Neubauer e espectrofotômetro, em nível de significância de 5\%.

À respeito da avaliação da concentração espermática determinada pelo espermiodensímetro, Palmer e Barth (2003) afirmaram não ser um bom substituto das outras técnicas, por ser subjetivo. Este atual trabalho, no entanto, contradiz estes autores por demonstrar semelhança de resultados aos da câmara de Neubauer e aos do espectrofotômetro, além de alta correlação entre eles, dependendo apenas de uma correta padronização.

A câmara de Neubauer é de alta acurácia, com baixo custo, porém de execução mais demorada e trabalhosa (ROUGE, 2002), o espectrofotômetro é altamente utilizado em centrais de inseminação artificial por ser de rápida execução porém seu custo é alto (FARRELL et al., 1998), enquanto o espermiodensímetro apresenta um custo intermediário, uma execução rápida, porém ainda requer padronização para evitar resultados equivocados no exame, além de ser de análise mais subjetiva (PALMER E BARTH, 2003).

De posse destas afirmativas e associando-se ao fato da diferença estatística obtida entre a câmara de Neubauer e o espectrofotômetro neste trabalho, bem como a semelhança de resultados do espermiodensímetro com a câmara de Neubauer e com o espectrofotômetro, pode-se considerar o primeiro como um método a ser amplamente utilizado a campo, por ser de execução mais rápida que o segundo, e de custo menor que o terceiro. 
BARBOSA, C.P., OLIVEIRA, W.C. e RIBEIRO JÚNIOR, W.R. Comparação entre as principais técnicas de análise da concentração espermática em exames andrológicos de touros a campo. PUBVET, Londrina, V. 5, N. 16, Ed. 163, Art. 1100, 2011.

\section{CONCLUSÃO}

A técnica de espermiodensitometria pode ser utilizada em análises da concentração espermática de touros, principalmente a campo, com maior rapidez que com a câmara de Neubauer e com a mesma segurança de resultados, desde que devidamente padronizada, a baixos custos.

AGRADECIMENTOS: Central Nova Índia Genética (Dr. João de Oliveira), Uberaba - MG.

\section{REFERÊNCIAS}

ASBIA - Associação Brasileira de Inseminação Artificial. Comunicação Técnica, 2009. Disponível em <www.asbia.org.br>. Acesso em 08/12/2010.

AYRES, M.; AYRES Jr., M.; AYRES, D. L.; SANTOS, A. S. Bioestat 2.0 - aplicações estatísticas nas áreas das ciências biológicas e médicas. Belém: Sociedade Civil Mamirauá; Brasília: CNPq, 2000. 272 p.

BRASIL, Produção da Pecuária Municipal 2009, v. 37, 2009, 55 p.

BRUNO, D. G.; NAMINDOME, A.; VIANNA, W. L.; ROSSETO, A. C.; RODRIGUES, P. H. M. Estudo comparativo de diferentes técnicas de mensuração da concentração espermática do ejaculado de reprodutores suínos. In: XI Congresso Brasileiro de Veterinários Especialistas em Suínos - 30/09 a 03/10 de 2003. Anais...

CHRISTENSEN, P.; STENVANG, J. P.; GODFREY, W. L. A flow cytometric method for rapid determination of sperm concentration and viability in mammalian and avian semen. Journal of Andrology, v. 25, n. 2, p. 255-264, mar-apr. 2004.

FARRELL, P. B.; PRESICCE, G. A.; BROCKETT, C. C.; FOOTE, R. H. Quantification of bull sperm characteristics measured by computer-assisted sperm analysis (CASA) and the relationship to fertility. Theriogenology, v. 49, n. 4, p. 871-879, mar. 1998.

MADEIRA, E.M.; BIANCHI, I.; CALDERAM, K.; MASCHIO, E.F.; CORRÊA, E.K.; PERONDI, A.; LUCIA JR., T.; COLARES, T.; CORRÊA, M.N. Comparação de diferentes métodos para avaliação da concentração de sêmen suíno. Disponível em < www.ufpel.edu.br/cic/2006/arquivos/CA-00354.rtf>, 2006. Acesso em 27/12/2010.

MURGAS, L.D.S.; LIMA, D.; ALVARENGA, A.L.N.; ZANGERONIMO, M.G.; OBERLENDER, G.; OLIVEIRA, S.L. Estudo comparativo de diferentes técnicas de avaliação da concentração espermática em suínos. Archivos de zootecnia, v. 59, n. 227, p. 463-466, 2010.

PALMER, C. W.; BARTH, A. D. Comparison of the BullMate sperm quality analyzer with conventional means of assessing the semen quality and breeding soundness of beef bulls. Animal Reproduction Science, v. 77, n. 3-4, p. 173-185, jul. 2003. 
PIMENTEL, C. A.; DESCHAMPS, J. C. Efeitos de produtos anabolizantes sobre a qualidade do sêmen de touros. Ciência Rural, Santa Maria, v. 25, n. 2, p. 265-269, 1995.

PIMENTEL, C. A. Infertilidade em Bovinos. In: RIET-CORREA. F.; SCHILD, A. L.; MÉNDEZ, M. del C. Doenças de Ruminantes e Eqüinos. Pelotas: Universitária - UFPel. Pelotas p.559590, 1998. 658 p.

ROUGE, M. Appearance of semen and sperm concentration. Disponível em /http: www.visionveterinaria.com/ em 30/11/2002. Acesso em 28/11/2010.

SUKCHAROEN, N.; NGEAMJIRAWAT, J.; CHANPRASIT, Y.; ARIBARG, A. A comparison of Makler counting chamber and improved Neubauer hemocytometer in sperm concentration measurement. Journal of Medical Association of Thailand, v. 77, n. 9, p. 471-476, sep. 1994.

TOMLINSON, M.; TURNER, J.; POWELL, G.; SAKKAS, D. One-step disposable chambers for sperm concentration and motility assessment: how do they compare with the World Health Organization's recommended methods? Human Reproduction, v. 16, n. 1, p. 121-124, jan. 2001.

VIANNA, W.L.; BRUNO, D.G.; NAMINDOME, A.; ROSSETO, A.C.; RODRIGUES, P.H.M.; PINESE, M.E.; MORETTI, A.S. Estudo comparativo da eficiência de diferentes técnicas de mensuração da concentração espermática em suínos. Revista Brasileira de Zootecnia, v. 33, n. 6, supl. 2, p. 2054-2059, dec. 2004. 